\title{
Local Strain Measurements at Dislocations, Disclinations and Domain Boundaries.
}

Martin Hÿtch ${ }^{1}$, Christophe Gatel ${ }^{1}$, Axel Lubk ${ }^{2}$, Thibaud Denneulin ${ }^{1}$, Lise Durand ${ }^{1}$, Nikolay Cherkashin ${ }^{1}$ and Etienne Snoeck ${ }^{1}$.

${ }^{1 .}$ CEMES-CNRS and Université de Toulouse, 29 rue Jeanne Marvig, 31055 Toulouse, France.

2. Institute of Structure Physics, Technische Universität Dresden, 01062 Dresden, Germany.

We present the state of the art in strain mapping at the nanoscale using aberration-corrected highresolution transmission electron microscopy, HRTEM and HAADF-STEM, and dark-field electron holography (DFEH) [1]. In particular, we will focus on the examination of localized strains around defects. A broader comparison of TEM strain mapping techniques can be found in a recent review [2].

High-resolution images can be analysed by geometric phase analysis (GPA) [3] or applying peak-finding routines to determine the positions of individual atomic columns. GPA is best adapted to measuring the deformation of the crystalline lattice, as the example concerning a five-fold twinned $\mathrm{Pt}$ nanoparticle will show (Figure 1). These star-shaped particles [4] have a disclination-like strain fields around their centres, as found for Au nanoparticles [5]. In multiferroic materials, peak-finding can be used to determine the relative displacements of atom columns within a unit cell [6]. The local polarization and strain can be mapped around dislocations and in the vicinity of domain walls (Figure 2).

DFEH was developed to measure strain over wide fields of view to high precision and nanometre spatial resolution. We have nevertheless been applying the technique to the study of quantum dots to relatively high resolution [7]. As with any TEM technique the samples are necessarily thin which allows some of the strain to be relaxed: the well-known thin film effect. In addition, dynamical scattering effects the strain information [8]. These issues will be addressed using a combination of finite-element modeling (FEM) and dynamical-scattering simulations.

This overview will, in addition, show results from the recently installed I2TEM microscope (Hitachi), an instrument specifically designed for DFEH experiments and aberration-corrected HRTEM over wide fields of view and for in-situ experiments (Figure 3).

[1] MJ Hÿtch, F Houdellier, F Hüe and E Snoeck, Nature 453 (2008), p. 1086.

[2] MJ Hÿtch and A Minor, MRS Bulletin 39 (2014), p. 139.

[3] MJ Hÿtch, E Snoeck and R Kilaas, Ultramicroscopy 74 (1998), p. 131.

[4] LM Lacroix, C Gatel, R Arenal et al, Angewandte Chemie-International Edition 51 (2012), p. 4690.

[5] CL Johnson et al, Nature Materials 7 (2008), p. 120.

[6] A Lubk et al, Nano Letters 13 (2013), p. 1410.

[7] N Cherkashin et al., Appl. Phys. Lett. 102 (2013), p. 173115.

[8] A Lubk et al, Ultramicroscopy 136 (2014) p. 42.

[9] The authors acknowledge financial support from the French National Agency (ANR) in the frame of its program in Nanosciences and Nanotechnologies (ANR-2011-PRGE-009-03 Multisolsi), the European Union under the Seventh Framework Programme under a contract for an Integrated Infrastructure Initiative Reference 312483-ESTEEM2 and the European Metrology Research 
Programme (EMRP) Project IND54 Nanostrain. The EMRP is jointly funded by the EMRP participating countries within EURAMET and the European Union.
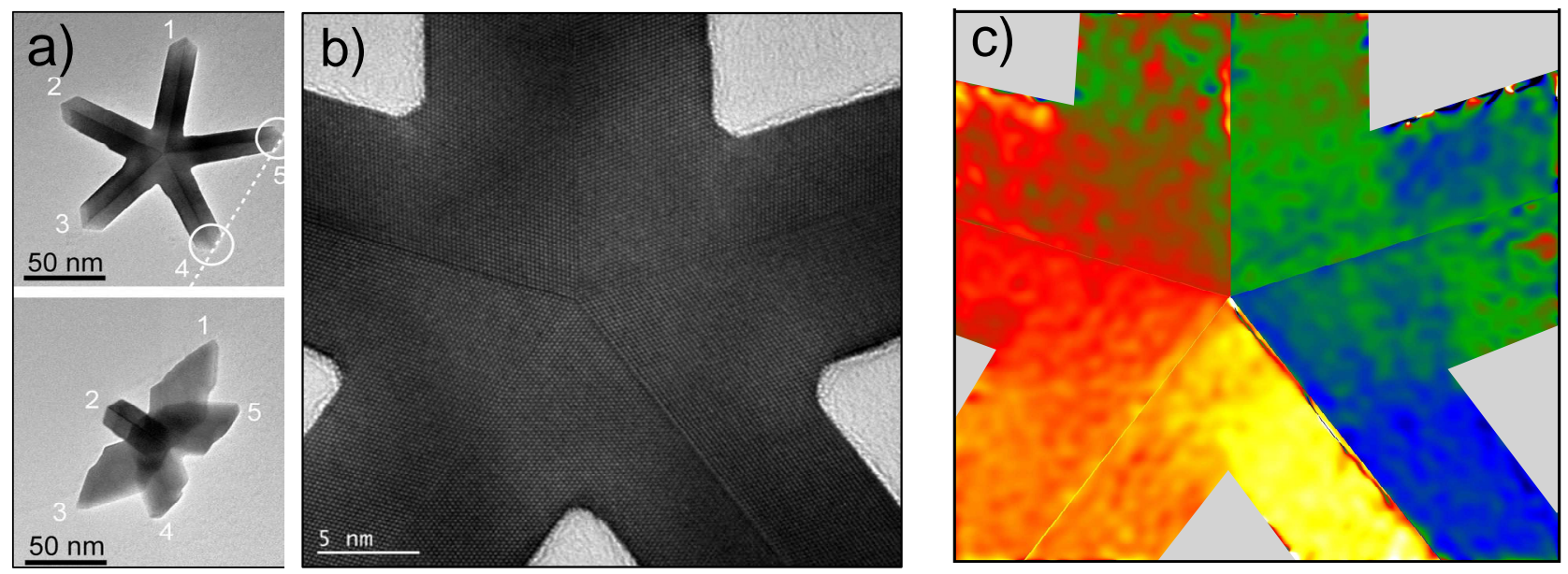

Figuře 1. Disclination in five-fold nanostars of Pt: (a) bright-field images of nanoparticle, in-plane and inclined; (b) aberration-corrected HRTEM image of nanoparticle core showing 5-fold twinning; (c) corresponding in-plane orientation map showing linearly increasing rotation around disclination core.
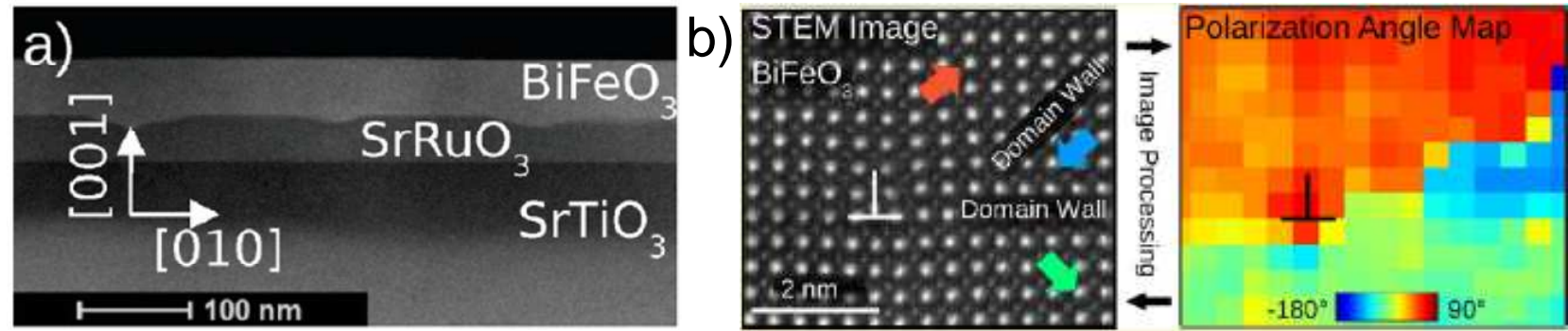

Figuře 2. Dislocations in $\mathrm{BiFeO}_{3}$ : (a) medium-resolution HAADF-STEM image of layer structure; (b) aberration-corrected atomic-resolution HAADF-STEM image of dislocation and domain boundary; (c) local polarization map determined from column displacements.

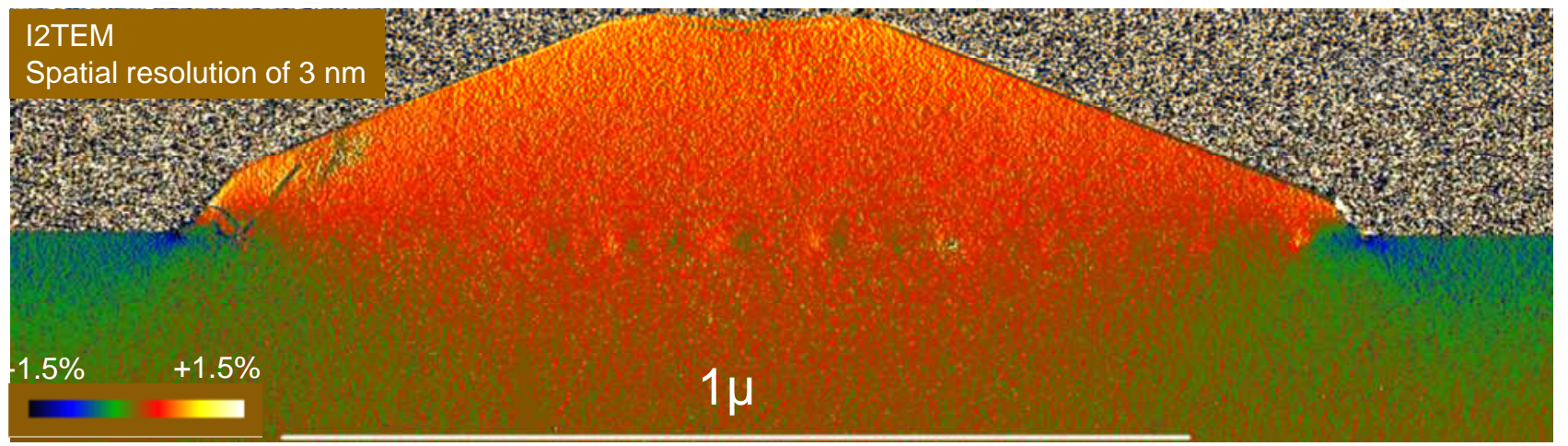

Figuře 3. Defects in strained-islands of $\mathrm{SiGe}$ on $\mathrm{Si}$ : horizontal deformation component mapped by DFEH on the I2TEM microscope with respect to silicon substrate. 